\title{
Pregnancy Management in NMO: A Case of MOG- Antibody Positive NMO and Literature Review
}

\section{Myintzu Min ${ }^{*}$, Patricia Caruana and Jeannette Lechner-Scott}

John Hunter Hospital, Department of Neurology, Newcastle, New South Wales, Australia

*Corresponding author: Myintzu Min, John Hunter Hospital, Department of Neurology, Newcastle, New South Wales, Australia, Tel: 02 4921 5577; E-mail: Myintzu.Min@hnehealth.nsw.gov.au

Received date: July 25, 2016; Accepted date: August 29, 2016; Published date: August 31, 2016

Copyright: @ $2016 \mathrm{Min} \mathrm{M}$, et al. This is an open-access article distributed under the terms of the Creative Commons Attribution License, which permits unrestricted use, distribution, and reproduction in any medium, provided the original author and source are credited.

\begin{abstract}
Neuromyelitis optica (NMO) is a rare but severely disabling disease, affecting predominantly the optic nerves and spinal cord. Aquaporin-4 (AQP4 lgG) antibodies have been described as characteristic and pathogenic for the disease. Some NMO cases are AQP4 lgG negative, of which a fraction has antibodies against myelin oligodendrocyte glycoprotein (MOG). We report the pregnancy of a 42 year old English woman who was diagnosed with neuromyelitis optica spectrum disorder (NMOSD), with anti-MOG antibodies. She fell pregnant prior to starting immunosuppressive therapy. At week 30 of gestation, she experienced a relapse and was treated with high dose steroids. She developed further right hand numbness in the week post-partum and had MRI confirmation of a new cervical lesion. This case suggests that unlike in multiple sclerosis (MS), pregnancy does not protect from relapses in NMO. Here we review the limited experience of pregnancy outcomes in anti-MOG antibodies positive NMO patients.
\end{abstract}

Keywords: Neuromyelitis optica spectrum disorder; Multiple sclerosis; Pregnancy

\section{Introduction}

NMO is a rare demyelinating disease of predominantly spinal cord and optic nerve. It was once thought to be a variant of MS, until the discovery of the autoantibody against AQP4 in 2004. This immunological evidence, together with clinical, radiological, and pathological studies, has reinforced $\mathrm{NMO}$ as a distinct astrocyte disease, separate from MS.

Monophasic NMO, originally Devic's classical syndrome, was first characterised as an acute disorder of transverse myelitis (TM) and optic neuritis $(\mathrm{ON})$ occurring simultaneously or in rapid succession [1]. Such cases are associated with a more equitable sex distribution, relatively younger age at disease onset, tendency to present with simultaneous myelitis and bilateral $\mathrm{ON}$, lower frequency of other autoimmune diseases, and lower prevalence of serum AQP4 IgG compared to relapsing NMO [2]. In more than $80-90 \%$ of cases, NMO follows a relapsing course, which is more commonly found in women and associated with older age at onset, longer time interval between index events, less severe motor impairment with the first myelitis attack, and with the presence of systemic autoimmunity [2].

The recent 2015 International Panel for NMO Diagnosis (IPND) has unified the traditional 2006 NMO definition with modern NMOSD definitions [3]. The new nomenclature is NMOSD, with or without AQP4 IgG. The presence of AQP4 IgG is no longer the key to the pathogenesis of this disorder, as they are not detectable in up to $40 \%$ of patients fulfilling the clinical criteria of NMO [4]. About $20-30 \%$ of AQP4 IgG negative cases have antibodies against MOG [4].

For the first time, the 2015 Wingerchuk criteria allow for NMOSD diagnosis in patients who have not experienced clinical involvement of either optic nerves or spinal cord. Not only are the criteria important in distinguishing AQP4-IgG negative NMOSD from MS, they will lead to appropriate immunotherapy for relapse prevention. This is critical as recent observational data suggest that interferon- $\beta$, natalizumab, and fingolimod may worsen NMO [5].

Despite the growing interest in NMOSD, epidemiological studies on NMOSD are sparse, probably because NMO is often misdiagnosed as MS. The incidence and prevalence rates of NMO in Australia and New Zealand are 0.37 per million and 0.70 per 100,000 respectively [6]. The epidemiology on anti-MOG antibodies NMOSD is even scarcer. NMO is more prevalent among non-Caucasians, and is three times more common in the Asian population. Unlike MS, it is not associated with a latitudinal gradient. The Australian and New Zealand clinical study between 2009 and 2012, documented 151 NMO cases, of which 8 were seronegative [6]. This study did not differentiate anti-MOG antibody positive cases. The 2015 Wingerchuk criteria will hopefully facilitate comparable epidemiologic studies in the future.

Up to $30 \%$ of AQP4 negative NMO patients have MOG antibodies, which accounts for $5 \%$ of the total NMO population. MOG is recognised as one of the autoantigens in paediatric MS and acute disseminated encephalomyelitis (ADEM) [2,7]. Its role in adult MS, is however controversial. In NMOSD patients, three separate groups have shown that no patient was positive for both MOG and AQP4 antibodies [7-9]. Using cell-based assay (CBA) with the full length MOG, two rare cases were reported with double positivity for AQP4 and MOG antibodies in a Spanish study [9]. Occasionally, patients without detectable serum AQP4 IgG are later found to be seropositive. There may be technical explanations in some cases. Jarius et al. reported a higher median serum AQP4 IgG titre during clinical relapses compared to remission and its potential influence by immunosuppressive therapy $[10,11]$. However, AQP4 IgG titres do not appear to be a reliable indicator of disease activity as persistent high levels can occur with disease quiescence [10]. 
Citation: Min M, Caruana P, Lechner-Scott J (2016) Pregnancy Management in NMO: A Case of MOG- Antibody Positive NMO and Literature Review. J Neurol Neurophysiol 7: 391. doi:10.4172/2155-9562.1000391

Page 2 of 4

There are characteristic disease patterns between the antibody groups. More severe acute $\mathrm{ON}$ and motor symptoms have been reported in the AQP4 positive group, in contrast to simultaneous myelitis and sensory symptoms in the AQP4 negative group [12]. Amongst the AQP4 negative patients with MOG positive antibodies, there is a trend towards non-female and non-Caucasian predominance, better prognosis, fewer relapses, and more favourable outcomes than the AQP4 positive patients [13]. A comparative study has shown a better recovery of Expanded Disability Status Scale (EDSS) scores from the onset episode in MOG positive patients, in contrast to AQP4 positive patients [8]. The MRI features in MOG positive cases had more involvement of the conus and had resemblance to ADEM. In addition, there is a lack of association with other autoimmune diseases, unlike AQP4 positive cases. Hence, recognition of MOG positive cases has important clinical and prognostic implications.

The pathogenesis and clinical course of MOG IgG associated NMOSD remains largely undefined, more so in pregnancy. This report describes a case of an anti-MOG antibody positive patient and her pregnancy, and reviews the current literature on pregnancy outcomes and management of anti-MOG and anti-AQP4-antibody NMO patients.

\section{Case Report}

Ms SR is a 42 year old artist and mother of a 9 year old daughter, who was diagnosed with NMOSD in May 2015, with anti-MOG antibody positive and AQP4 IgG negative results. Co-morbidities include mild asthma and a previous history of depression. Prior to her diagnosis, she had undergone extensive spinal surgeries from 2008 to 2014, for presumed radiculopathies. Unfortunately, images preceding 2012 were unable to be retrieved to confirm absence of myelitis at this time point.

Her first neurological symptom started with left leg numbness, weakness, and bowel and bladder dysfunction in 2008. They were attributed to a L5/S1 disc rupture which she underwent surgery with improvement. In 2012, she developed left arm numbness that improved after a C5 to C7 anterior fusion. In 2014, she experienced sensory abnormalities in her right arm, followed by transient symptoms suggestive of optic neuritis (ON). She was diagnosed with recurrent myelitis from 2014, with episodes of lower limbs sensory disturbance, bowels and bladder symptoms. On average, she experienced two relapses a year since 2014. Residual examination findings include mild right arm and leg numbness and lower limb hyperreflexia. Ms SR has normal visual acuity, cranial nerves, full strength and no cerebellar signs.

Serial MRIs from 2014 to 2015 indicated progression of demyelinating lesions from C 3 to C5 cord levels. During one relapse in April 2015, there was a larger area of demyelination in the right hemicord of $\mathrm{C} 3$, with contrast enhancement, confirming active disease. She had no cerebrospinal fluid (CSF) restricted oligoclonal IgG bands, and no pleocytosis (only 2 white cells and 1 red cell). IgG index and protein levels were within normal limits in her CSF. Serology for antinuclear antibodies (ANA), anti-double-stranded DNA, extractable nuclear antigen (ENA) and anti-neutrophil cytoplasmic antibody (ANCA) were negative. NMDA receptor and AQP4 IgG antibodies were not detectable when tested during a clinical relapse in 2014. AntiMOG antibodies were positive, when available for testing in 2015. She was treated on three occasions with intravenous pulsed methylprednisolone with good response.

Ms SR fell pregnant with her second child in 2015 and was therefore, not as planned, commenced on immunotherapy. She was monitored closely in the Neuro-immunology Clinic, in conjunction with her Obstetrician.

At week 30 of gestation, Ms SR developed a NMO relapse with increasing numbness and tingling in both arms and legs, especially on her right side. She underwent a 3 day course of intravenous steroids with good effects. She delivered a healthy baby girl on 4 April 2016, but developed increasing right hand numbness in the week post-partum. MRI showed a new T2 lesion at C2. She received her first rituximab infusion on 19 April 2016 (Figures 1-3).

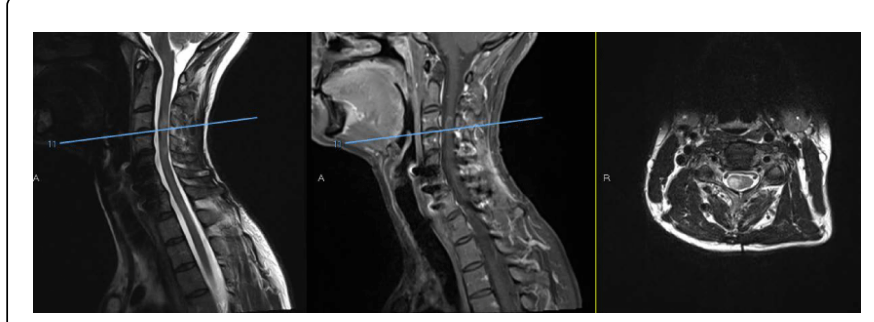

Figure 1 (Left to right): MRI T2 weighted sagittal of the cervical spine with increased signals; MRI T1 sagittal and axial sequences showing contrast enhancement and extending area of demyelination in C3 during a relapse in April 2015.

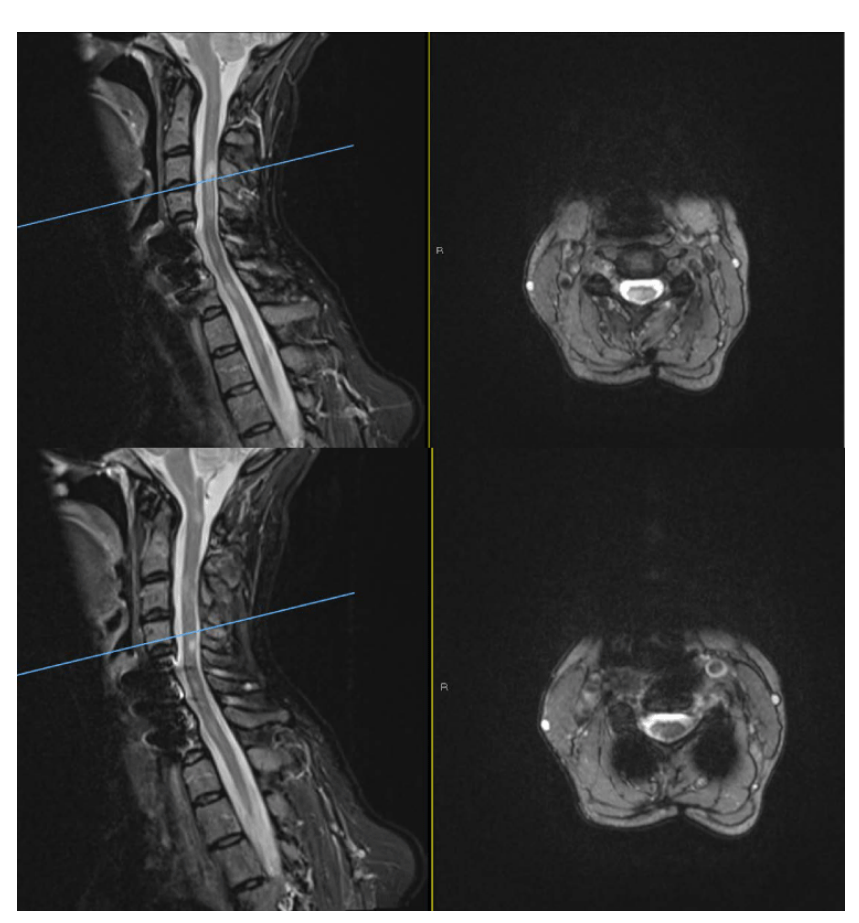

Figure 2: MRI sagittal T2/STIR and axial T2 sequences of the cervical spine showing a new $\mathrm{C} 2$ lesion at post-partum. C3/4 level lesion remains stable. Characterisation of $\mathrm{C} 4$ to $\mathrm{C} 7$ is limited by artefact from the implanted plate and screws. 


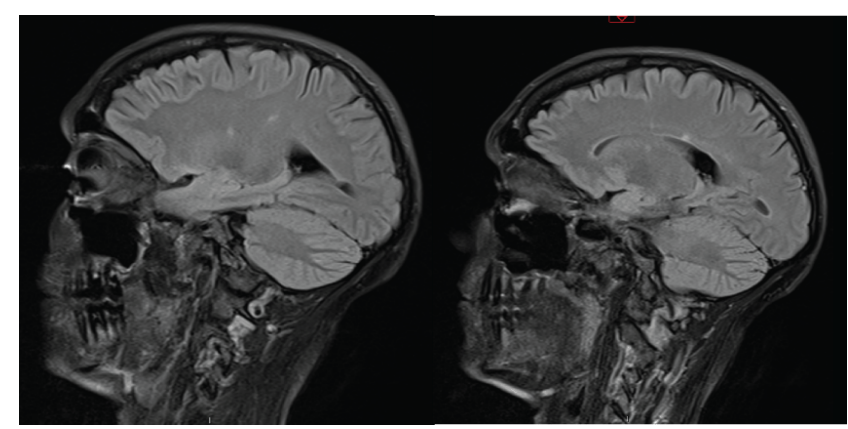

Figure 3: MRI sagittal FLAIR sequences showing non-specific hyperintensities in the pericallosal white matter of the right hemisphere.

\section{Discussion}

NMO has a striking female preponderance in Australia, with a 6:1 female to male ratio [6]. The diagnosis is commonly made in women of childbearing ages. However, there are no prospective studies reviewing the effects of pregnancy on the course of NMO. Until recently, experimental and clinical reports have demonstrated the presence of AQP4 in human and animal placenta, and have linked AQP4-mediated placental inflammation to fetal death [14].

Retrospective reviews from Europe and East Asian populations by Bourre et al. and Nour et al. have indicated that the annualized relapse rate (ARR) of NMO is increased in the third trimester of pregnancy $[15,16]$. Bourre et al. demonstrated the increase ARR in the third trimester and first 3 months post-partum, by comparing 13 pregnancies in their NMOSD cohort with MS patients from the PRIMS study. No significant decrease in ARR during pregnancy was found in both Japanese and Malaysian cohorts, 12 and 7 pregnancies, respectively $[17,18]$. Slightly larger studies by Kim et al. and Fragoso et al. reported no difference in ARR before and during pregnancy, in their review of 26 and 17 pregnancies, respectively [19,20]. Considering the diversity of ethnic and genetic differences from the studies, all results were consistent with an increase in ARR in the 3 months post-partum. Pregnancy in patients with MS also demonstrates this pattern of increased relapse rate post-partum. However, the severity of these relapses and the accumulated disability after the patient become pregnant seem worse in NMO.

The potential influence of immunomodulatory and immunosuppressive treatments before and during pregnancy play a part in these studies. In general recommendation has been to continue immunosuppression during pregnancies based on the Japanese study reporting that those pregnant women who were on immunosuppressants (azathioprine, tacrolimus) and low-dose prednisolone, or higher dose of prednisolone alone had no relapse, while those who were on low dose prednisolone only were associated with relapse. Nonetheless, the 8 pregnancies in Nour et al. that occurred on immunosuppressants of prednisolone, azathioprine or a combination, still resulted in 6 miscarriages. The Malaysian study, apart from short course steroids for relapses, tried to examine the natural history of 7 pregnancies that occurred without immunosuppressants. They reported a high ARR post-partum. All children from the Malaysian pregnancy study were born healthy, and none developed NMOSD. Two German case studies reported that exposure to rituximab in pregnancy was not associated with adverse events for both mother and infant [21,22]. Clearly, there is a lack of treatment guidelines on the recommended preventative therapies during pregnancy, given the variable protocols in the studies.

Current NMO treatments include corticosteroids and plasma exchange, which reduce the severity of acute attacks. Preventative treatment like azathioprine and rituximab are at present level IV evidence for first-line therapy in NMO management. It remains difficult to draw conclusions about the safety of azathioprine plus lowdose prednisolone or rituximab during the course of NMO pregnancy, based on existing literature.

Patients included in the above studies are, notably, all AQP4 IgG positive NMOSD women. There are no studies or case reports reviewing pregnancy outcomes in AQP4 negative, MOG positive NMO patients. Our case demonstrated a relapse in her third trimester and a new T2 lesion post-partum without preventative treatment, reflecting high disease activity in the third trimester and post-partum.

\section{Conclusion}

The literature about pregnancy outcomes in NMO is limited and only includes, so far, AQP4 positive cases. MOG positive NMO has only recently been described and experience is slowly accumulating. One should be vigilant about testing of anti-MOG antibodies in AQP4 negative $\mathrm{NMO}$ cases for diagnostic and prognostic reasons.

The risk of relapse in a MOG positive NMO patient during pregnancy remains undefined. Our case study suggests that pregnancy does not protect from relapses, consistent with most AQP4 positive NMO pregnancy studies.

The benefit and risk of preventative treatment during pregnancy or immediately after delivery in NMO patients should be discussed with the patient, due to the higher risk of relapse post-partum, regardless of AQP4 positivity.

The influence of anti-MOG antibodies status on pregnancy is unknown and registries are required given the rareness of the disease and its unfavourable prognosis.

\section{References}

1. Pereira WL, Reiche EM, Kallaur AP, Kaimen-Maciel DR (2015) Epidemiological, clinical and immunological characteristics of neuromyelitis optica: A review. J Neurol Sci 355: 7-17.

2. Jasiak-Zatonska M, Kalinowska-Lyszczarz, Michalak S, Kozubski W (2016) The immunology of NMO-current knowledge, clinical implications, controversies and future perspectives. Int J Mol Sci 17: 273.

3. Wingerchuk DM, Banwell B, Bennett JL, Cabre P, Carroll W, et al. (2015) International consensus diagnostic criteria for neuromyelitis optica spectrum disorders. Neurology 85:177-189.

4. Pröbstel AK, Rudolf G, Dornmair K, Collongues N, Chanson JB, et al. (2015) Anti-MOG antibodies are present in a subgroup of patients with a neuromyelitis optica phenotype. J Neuroinflammation 12: 46.

5. Kowarik MC, Soltys J, Bennett JL (2014) The treatment of neuromyelitis optica. J Neuroophthalmol 34: 70-82.

6. Broadley S, Barnett MH, Kermode AG, Marriott M, Parratt J, et al. (2016) Incidence and prevalence of NMO in Australia and New Zealand: A population-based, clinical survey. ANZ NMO Epidemiology.

7. Kitley J, Waters P, Woodhall M, Leite MI, Murchison A, et al. (2014) Neuromyelitis optica spectrum disorders with aquaporin-4 and myelinoligodendrocyte glycoprotein antibodies: A comparative study. JAMA Neurol 71: 276-283. 
Citation: Min M, Caruana P, Lechner-Scott J (2016) Pregnancy Management in NMO: A Case of MOG- Antibody Positive NMO and Literature Review. J Neurol Neurophysiol 7: 391. doi:10.4172/2155-9562.1000391

Page 4 of 4

8. Sato DK, Callegaro D, Lana-Peixoto MA, Waters PJ, de Haidar Jorge FM et al. (2014) Distinction between MOG antibody-positive and AQP4 antibody-positive NMO spectrum disorders. Neurology 82: 474-481.

9. Höftberger R, Sepulveda M, Armangue T, Blanco Y, Rostásy K, et al. (2015) Antibodies to MOG and AQP4 in adults with neuromyelitis optica and suspected limited forms of the disease. Mult Scler 21: 866-874.

10. Jarius S, Franciotta D, Paul F, Ruprecht K, Bergamaschi R, et al. (2010) Cerebrospinal fluid antibodies to aquaporin-4 in neuromyelitis optica and related disorders: frequency, origin and diagnostic relevance. J Neuroinflammation 7: 52 .

11. Jarius S, Aboul-Enein F, Waters P, Kuenz B, Hauser A, et al. (2008) Antibody to aquaporin-4 in the long-term course of neuromyelitis optica. Brain 131: 3072-3080.

12. Di Pauli F1, Mader S, Rostasy K, Schanda K, Bajer-Kornek B, et al. (2011) Temporal dynamics of anti-MOG antibodies in CNS demyelinating diseases. Clin Immunol 138: 247-254.

13. Jarius S, Ruprecht K, Wildemann B, Kuempfel T, Ringelstein M, et al. (2012) Contrasting disease patterns in seropositive and seronegative neuromyelitis optica: A multicentre study of 175 patients. J Neuroinflammation 9: 14.

14. Saadoun S, Waters P, Leite MI, Bennett JL, Vincent A, et al. (2013) Neuromyelitis optica IgG causes placental inflammation and fetal death. J Immunol 191: 2999-3005.

15. Bourre B, Marignier R, Zéphir H, Papeix C, Brassat D, et al. (2012) Neuromyelitis optica and pregnancy. Neurology 78: 875-879.
16. Nour MM, Nakashima I, Coutinho E, Woodhall M, Sousa F, et al. (2016) Pregnancy outcomes in aquaporin-4-positive neuromyelitis optica spectrum disorder. Neurology 86: 79-87.

17. Shimizu Y, Fujihara K, Ohashi T, Nakashima I, Yokoyama K, et al. (2015) Pregnancy-related relapse risk factors in women with anti-AQP4 antibody positivity and neuromyelitis optica spectrum disorder. Mult Scler .

18. Hor JY, Chow HB, Lim TT, Chia YK, Tan K, et al. (2016) A natural history study of the effect of pregnancy on AQP4 antibody-positive NMOSD. Mult Scler 22: 425.

19. Kim W, Kim SH, Nakashima I, Takai Y, Fujihara K, et al. (2012) Influence of pregnancy on neuromyelitis optica spectrum disorder. Neurology 78: 1264-1267.

20. Fragoso YD, Adoni T, Bichuetti DB, Brooks JB, Ferreira ML, et al. (2013) Neuromyelitis optica and pregnancy. J Neurol 260: 2614-2619.

21. Ringelstein M, Harmel J, Distelmaier F, Ingwersen J, Menge $T$, et al. (2013) Neuromyelitis optica and pregnancy during therapeutic B cell depletion: Infant exposure to anti-AQP4 antibody and prevention of rebound relapses with low-dose rituximab postpartum. Mult Scler. 19: 1544-1547.

22. Pellkofer HL, Suessmair C, Schulze A, Hohlfeld R, Kuempfel T (2009) Course of neuromyelitis optica during inadvertent pregnancy in a patient treated with rituximab. Mult Scler 15: 1006-1008. 